\title{
Wetlands construídos para o tratamento de esgoto doméstico: uma análise comparativa do custo do ciclo de vida
}

\author{
Constructed wetlands for treating domestic wastewater: \\ a comparative analysis of life cycle costs
}

\section{Taise Perondi \\ Delmira Beatriz Wolff \\ Samara Terezinha Decezaro \\ Ronaldo Kanopf de Araújo}

\section{Resumo}

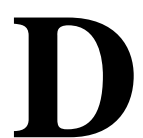

evido à carência de saneamento básico no Brasil, muitas habitações realizam o tratamento descentralizado de esgotos sanitários. Assim, estudos de custo das alternativas de tratamento são importantes.

Uma análise do custo do ciclo de vida foi realizada para investigar o custo dos sistemas wetlands construídos para o tratamento de esgoto sanitário, comparando três cenários com as configurações mais comuns utilizadas. O cenário 1 foi constituído por uma ETE em escala real, localizada no sul do Brasil, composta de um tanque séptico e de um WCFV com recirculação ao tanque séptico. Os demais cenários foram criados: o cenário 2 foi formado por um WCFV, e o cenário 3 foi composto de um tanque séptico, um WCFV e um WCFH. Os valores presentes líquidos obtidos pela análise do custo do ciclo de vida para os cenários 1,2 e 3 foram decrescentes. O cenário 3 apresentou menor custo do ciclo de vida. O cenário 1 foi o pior em relação à análise, entretanto, devido à utilização da recirculação, este apresentou melhor desempenho ambiental. A análise do custo do ciclo de vida, por levar em conta os custos ao longo da vida útil, mostrou-se uma ferramenta importante e adequada para a escolha da forma de tratamento de menor custo.

Palavras-chave: Tratamento descentralizado. Ferramenta de gestão. Custos de sistema wetland construído. Custo do ciclo de vida.

${ }^{1}$ Taise Perondi ${ }^{1}$ Universidade Tecnológica Federal do Paraná Frederico Beltrão - PR - Brasil

${ }^{2}$ Delmira Beatriz Wolff ${ }^{2}$ Universidade Federal de Santa Maria Santa Maria - RS - Brasil

${ }^{3}$ Samara Terezinha Decezaro ${ }^{3}$ Universidade Federal de Santa Maria Frederico Westphalen - RS - Brasil

${ }^{4}$ Ronaldo Kanopf de Araújo ${ }^{4}$ Universidade Federal de Santa Maria Santa Maria - RS - Brasil

Recebido em 23/02/19 Aceito em 12/07/19

\section{Abstract}

Due to the lack of basic sanitation in Brazil, many households use decentralised treatment of their wastewater. Therefore it is important to analyse the costs of wastewater treatment alternatives. A life cycle cost assessment was performed to evaluate the cost of constructed wetlands for wastewater treatment, comparing three scenarios with the most commonly used configurations. Scenario 1 consisted of a real scale wastewater treatment plant composed of a septic tank and a vertical flow constructed wetland with effluent recirculation to a septic tank, located in south of Brazil. The other two scenarios were created: scenario 2 was formed by a vertical flow constructed wetland and scenario 3 consisted of a septic tank, a vertical flow constructed wetland and a horizontal flow constructed wetland. The results showed that the net present value of the life cycle cost were decreasing for scenarios 1, 2 and 3. Scenario 3 presented lower life cycle cost. Scenario 1 had the poorest performance in the analysis, however, due to the recirculation of the wastewater this was the best performance in environmental terms. Due to the fact that life cycle cost analysis takes into account the cost throughout the life cycle of each scenario, it proved to be an important and suitable tool to help choosing the lowest cost treatment type.

Keywords: Decentralised treatment. Management tools. Costs of constructed wetland system. Life cycle cost.

PERONDI, T.; WOLFF, D. B.; DECEZARO, S. T.; ARAÚJ O, R. K. de. Wetlands construídos para o tratamento de esgoto doméstico: uma análise comparativa do custo do ciclo de vida. Ambiente Construído, Porto Alegre, v. 20, n. 2, p. 175189, abr./jun. 2020.

ISSN 1678-8621 Associação Nacional de Tecnologia do Ambiente Construído. http:/ / dx. doi. org/ 10.1590/ s1678-86212020000200394 


\section{Introdução}

No Brasil somente 52,36\% dos municípios são atendidos por rede coletora de esgotamento sanitário, e deste total de esgoto coletado $46 \%$ recebem algum tipo de tratamento anterior ao lançamento em um corpo hídrico (SISTEMA..., 2017). Reconhecendo a importância do setor do saneamento para a garantia da saúde dos indivíduos, as regiões desprovidas de um sistema de esgoto adequado, como áreas rurais e municípios de pequeno porte, precisam de alternativas viáveis para o tratamento individual de seus efluentes. Os wetlands construídos são sistemas descentralizados com relativamente baixos custos de operação e manutenção, simplicidade operacional e satisfatórios índices de remoção de poluentes. Há diversos trabalhos com bons resultados reportados no tratamento de esgoto doméstico para essa tecnologia no Brasil, como Platzer, Hoffmann e Cardia (2007), Cota, Von Sperling e Penido (2011), Lana et al. (2013), Sezerino et al. (2014), Horn et al. (2014), Trein et al. (2015), Von Sperling (2015) e Santos et al. (2016). Os sistemas wetlands construídos são ambientes constituídos por plantas aquáticas, micro-organismos e o meio filtrante (areia, cascalho ou outro material inerte), que se encontra saturado ou parcialmente saturado. São também sistemas flexíveis na operação, ou seja, permitem que sejam controladas algumas características, como o fluxo empregado e o tempo de detenção hidráulica. Dessa forma, ocorre bom desempenho na remoção de contaminantes como os compostos orgânicos, sólidos em suspensão, nitrogênio, fósforo, metais e patógenos (VYMAZAL, 2007). Além disso, trata-se de sistemas eficazes no tratamento de diversas águas residuárias, tais como esgoto sanitário e efluentes industriais (CHEN et al., 2006; CHANG et al., 2012; SEZERINO et al., 2012a, 2012b; PELISSARI et al., 2013; LANA et al., 2013; ROUSSO; PELISSARI; SEZERINO, 2015), águas pluviais (ARIAS; BERTRAND-KRAJEWSKI; MOLLE, 2014), águas residuárias da pecuária (PENG et al., 2012; PELISSARI, 2014) e lixiviados de aterros sanitários (VYMAZAL, 2009; WU et al., 2014; SEZERINO et al., 2014; SCHOLZ, 2016).

Wetland construído de fluxo vertical (WCFV) é uma configuração em que o efluente é disposto de forma intermitente e homogênea na superfície do filtro, o qual percola verticalmente através do material filtrante e, posteriormente, é coletado no fundo por meio da tubulação de drenagem/coleta (VYMAZAL; KRÖPFELOVÁ, 2009). Essa forma de aplicação do efluente promove uma elevada transferência de oxigênio atmosférico ao interior do sistema. No WCFV a demanda por área é inferior aos wetlands construídos de fluxo horizontal porque a distribuição do efluente ocorre em maior área devido à verticalidade da infiltração e porque o volume do filtro é mais bem aproveitado (SILVEIRA, 2015). Entretanto, esses sistemas não retêm quantidades significativas de fósforo, nem realizam a desnitrificação (COOPER et al., 1996).

Os custos envolvidos no sistema wetland construído se referem a sua implantação, operação e manutenção. Não há um custo padrão para esse sistema, que é variável de acordo com diversos fatores, tais como configuração do sistema, área a ser ocupada, região da implantação, macrófita utilizada e valor da energia local. Contudo, em comparação aos demais sistemas de tratamento de águas residuárias de semelhante eficiência, o wetland construído se destaca por seu reduzido custo de manutenção e operação (KADLEC; WALLACE, 2009). Os custos da operação e manutenção dos sistemas wetlands, em geral, são baixos. Para a operação desses sistemas é importante que seja realizado o monitoramento dos parâmetros qualitativos para observar se a estação está alcançando a eficiência de tratamento desejada. Também há os gastos operacionais devido ao bombeamento do efluente, porém o gasto energético desse processo é mínimo em comparação às outras formas de tratamento mecanizado convencionais. Segundo Brix (1999), o gasto energético em wetlands de fluxo subsuperficial é inferior a $0,1 \mathrm{~kW} \mathrm{~h} \mathrm{~m}^{-3}$ de efluente.

Segundo Thierry Jacquet, paisagista fundador da empresa francesa Phytorestore, que detém patentes desse sistema em vários países, inclusive no Brasil, o custo de implantação do sistema é similar ao custo de implantação de um projeto de paisagismo decorativo, sendo, porém, 30\% mais baixo do que o custo de implantação de uma unidade convencional de tratamento de água. Quanto ao custo de funcionamento, corresponde a $10 \%$ a $20 \%$ do custo de uma estação convencional e apresenta baixo consumo elétrico, não utilizando produtos químicos e sendo de simples manutenção (JARDINS..., 2011). Rai et al. (2013), que apontam a ampliação de utilização do sistema nas últimas décadas, reforçam esse aspecto ao identificarem que ele se mostra atrativo devido ao menor custo de implantação, operação e manutenção em relação a sistemas de tratamento tradicionais.

O custo do ciclo de vida (CCV) em um projeto é o valor total gasto com a implantação, operação, manutenção e desativação. A análise do custo do ciclo de vida (ACCV) é uma metodologia de estimativa de custos que contempla os possíveis custos envolvidos ao longo da vida útil de um projeto e resulta em um valor global dele (GLUCH; BAUMANN, 2004). Dessa forma, a ferramenta de ACCV pode ser útil na escolha de um arranjo tecnológico ou de uma tecnologia de tratamento de águas residuárias. Uma vez que não existe um 
custo padrão para a construção de sistemas do tipo wetland construído, estudos que utilizem ferramentas de avaliação das diferentes configurações operacionais são fundamentais. Em relação ao projeto de wetland construído, os custos de operação geralmente são inferiores aos de outras tecnologias de tratamento de águas residuárias. Como consequência, também a análise do CCV, representado pelo valor da implantação, manutenção e operação, é inferior em relação a outras formas mecanizadas de tratamento (KADLEC; WALLACE, 2009). Entretanto, poucos foram os estudos que buscaram realizar essa análise em wetland construído (CORBELLA; PUIGAGUT; GARFÍ, 2017; DIMURO et al., 2014; PUIGAGUTA et al., 2007). Assim, este estudo realiza a ACCV de sistemas wetland construídos para o tratamento de esgoto sanitário, tendo sido comparados três cenários, que incluíram as configurações mais comuns dos wetlands construídos para o tratamento de esgoto sanitário, de fluxo subsuperficial vertical e horizontal.

\section{Material e métodos}

Neste estudo foram comparados três cenários distintos, sendo o cenário 1 real, e os cenários 2 e 3 fictícios, sendo consideradas diferentes configurações para o tratamento de esgoto. No cenário 1 foi utilizada uma estação de tratamento de esgoto (ETE) doméstico constituída por tanque séptico (TS) e wetland construído de fluxo vertical (WCFV) com recirculação do efluente ao TS. No cenário 2 foi simulada a utilização de um wetland construído de fluxo vertical (WCFV). No cenário 3 foi simulada a utilização de um tanque séptico, um wetland construído de fluxo vertical (WCFV) e um wetland construído de fluxo horizontal (WCFH).

\section{Cenário 1: estação de tratamento de esgoto experimental}

A ETE experimental está localizada no câmpus da Universidade Federal de Santa Maria e é composta de tratamento primário por TS e tratamento secundário e avançado por WCFV com reciclo de efluente nitrificado ao TS (Figuras 1 e 2). A ETE foi dimensionada para o tratamento de efluentes domésticos provenientes da Casa do Estudante Universitário, para 10 habitantes, com vazão de projeto de $1.500 \mathrm{~L} \mathrm{~d}^{-1}$ e recirculação de até $100 \%$ do efluente tratado ao tanque séptico. Essa recirculação do efluente tratado foi proposta como estratégia para aumentar a eficiência de WCFV na remoção de nitrogênio e matéria orgânica carbonácea. A recirculação faz aumentar o tempo de percolação e fornece entrada de oxigênio adicional por convecção (PLATZER, 1999).

O tanque séptico circular foi construído abaixo do nível do solo, dimensionado de acordo com a NBR 7229 (ABNT, 1993). Considerou-se uma habitação unifamiliar com 10 habitantes (N=10), uma contribuição de

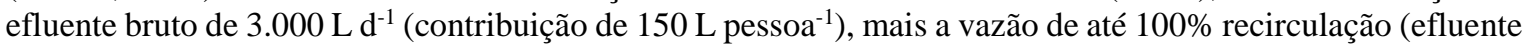
tratado no WCFV que retorna ao TS), uma contribuição de lodo fresco (Lf) de $1 \mathrm{~L}_{\text {pessoa }}{ }^{-1} \mathrm{~d}^{-1}$, tempo de detenção $(\mathrm{T})$ de 1 dia, intervalo de limpeza de 1 ano e taxa de acumulação total de lodo $(\mathrm{K})$ de 65 dias. Dessa forma, o TS foi projetado para um volume útil total de 4,65 $\mathrm{m}^{3}$. As dimensões resultantes foram: profundidade útil = 1,5 m; diâmetro = 2,0 m; e $\mathrm{A}=3,39 \mathrm{~m}^{3}$.

O WCFV foi dimensionado para uma taxa de aplicação hidráulica (TAH) de $120 \mathrm{~mm} \mathrm{~d}^{-1}$, considerando a aplicação de $100 \%$ de recirculação. Dessa forma, para atender a uma vazão de esgoto bruto de $1.500 \mathrm{~L} \mathrm{~d}^{-1}$ (equivalente a 10 pessoas, com contribuição de $150 \mathrm{~L}_{\text {pessoa}}{ }^{-1} \mathrm{~d}^{-1}$ ) mais um cenário de $1.500 \mathrm{~L} \mathrm{~d}^{-1}$ de recirculação (100\%), a vazão afluente ao WCFV deve ser de aproximadamente $3.000 \mathrm{~L} \mathrm{~d}^{-1}$. Para essa vazão e uma TAH de $120 \mathrm{~mm} \mathrm{~d}^{-1}$, a área superficial necessária é de $25 \mathrm{~m}^{2}$. Como a relação comprimento/largura adotada foi igual a 2, as dimensões resultantes do WCFV foram $7 \mathrm{~m}$ de comprimento por 3,5 m de largura, ou seja, área superficial de $24,5 \mathrm{~m}^{2}$. A altura total foi de $1,15 \mathrm{~m}$. As laterais e o fundo do WCFV foram impermeabilizados com manta asfáltica. O dimensionamento foi realizado somando-se as entradas por convecção e difusão ao transporte de oxigênio via macrófitas (0,02 $\mathrm{g} \mathrm{O}_{2} \mathrm{~m}^{-2} \mathrm{~d}^{-1}$ (KADLEC; WALLACE, 2009). A concentração de $\mathrm{O}_{2}$ obtida nesse cálculo deve ser maior que a demanda, que é de aproximadamente 4,3 $\mathrm{mg} \mathrm{O}_{2} \mathrm{mg}^{-1} \mathrm{~N}^{-\mathrm{NH}_{4}}{ }^{+}$oxidada (PLATZER, 1999).

O WCFV possui profundidade do leito filtrante de $0,75 \mathrm{~m}$. Foram preenchidas três camadas de brita: uma camada de brita 2 no fundo de $0,20 \mathrm{~m}$, uma camada filtrante média de brita 1 de $0,50 \mathrm{~m}\left(\mathrm{~d}_{10}=9,5 \mathrm{~mm}, \mathrm{~d}_{30}=\right.$ $12,5 \mathrm{~mm}, \mathrm{~d}_{60}=15 \mathrm{~mm}$; coeficiente de uniformidade = 1,58) e uma camada de fundo de $0,05 \mathrm{~m}$. Os parâmetros $\mathrm{d}_{10}$ e $\mathrm{d}_{60}$ são diâmetros efetivos do meio filtrante ( $\mathrm{dn}$ = diâmetro dos grãos do meio filtrante em milímetros, tal que n, em percentagem, dos grãos seja menor que aquele, em termos de massa). A planta utilizada no WCFV foi a macrófita Heliconia psittacorum, sendo realizado o transplantio de mudas com densidade de 7,7 mudas $\mathrm{m}^{2}$ (3 indivíduos por muda).

Em consulta a outros estudos buscaram-se alternativas de baixo custo a fim de reduzir a totalidade dos custos envolvidos no ciclo de vida de um wetland construído (CHEN et al., 2006; CHANG et al., 2012; SEZERINO 
et al., 2012a, 2012b; LANA et al., 2013; ROUSSO; PELISSARI; SEZERINO, 2015). Com base nessa etapa, os demais cenários foram elaborados e apresentaram desempenho semelhante ao cenário 1 (ETE experimental). Os três cenários propõem configurações para a satisfatória remoção da matéria orgânica carbonácea, sólidos e, também, nitrogênio.

\section{Figura 1 - Esquema da ETE experimental na Casa do Estudante Universitário da UFSM}

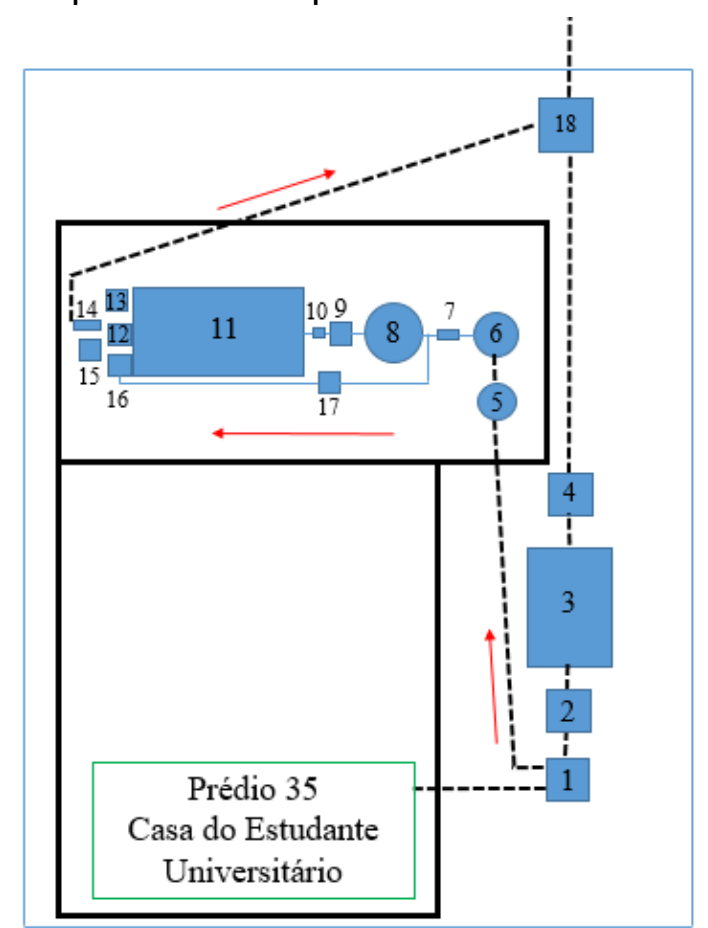

1 Caixa de lançamento de efluentes

2 Caixa de passagem

3 Tanque séptico

4 Caixa de passagem

5 Reservatório de efluentes

6 Tanque de equalização com bomba submersível

7 Canal com medidor de vazão

8 Tanque séptico

9 Bomba centrífuga

10 Medidor de vazão na tubulação

11 Wetland construído

12 Caixa de lançamento de efluentes

13 Bomba centrífuga

14 Canal com medidor de vazão

15 Reservatório de efluentes

16 Bomba centrífuga

17 Medidor de vazão na tubulação

18 Caixa de passagem para disposição final

$\longrightarrow$ Sentido do fluxo

Fonte: Araújo (2018)

\section{Figura 2 - ETE experimental instalada na Casa do Estudante Universitário da UFSM}

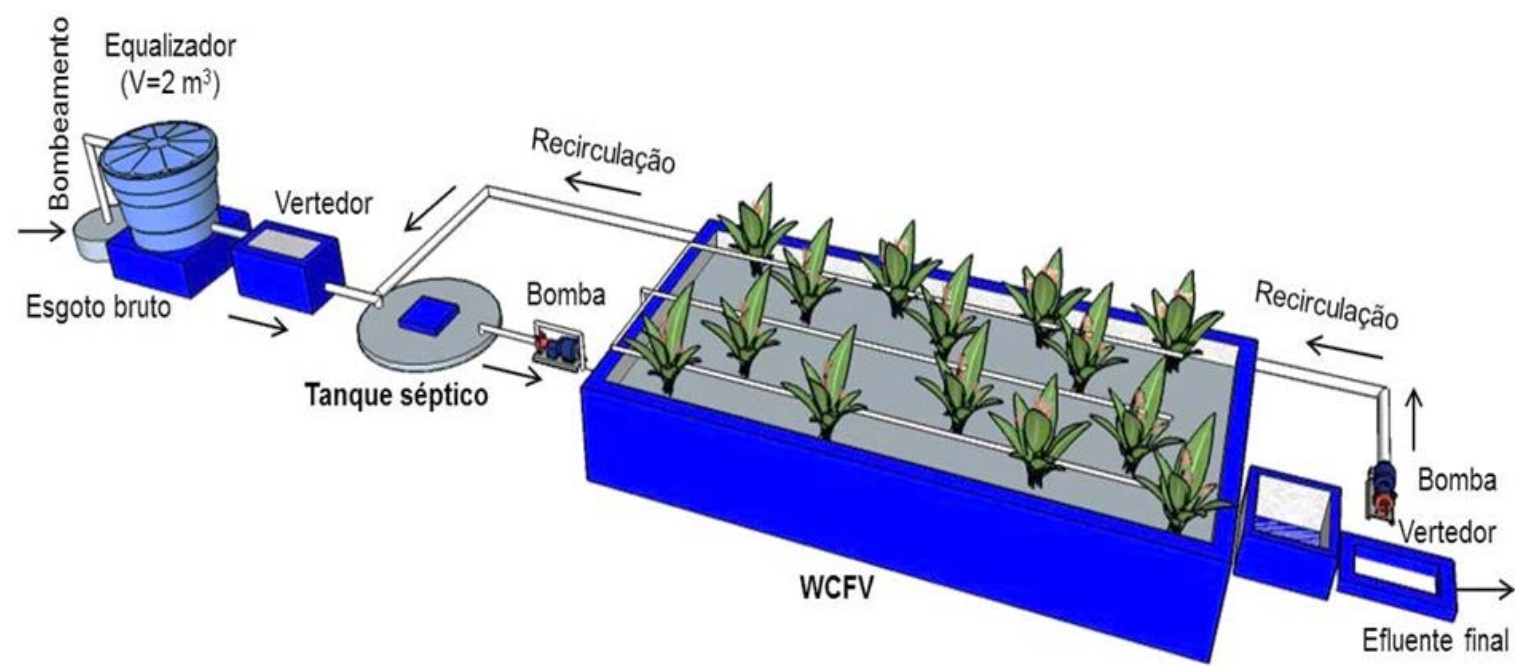

Fonte: Decezaro et al. (2018). 


\section{Cenário 2: ETE fictícia com outra planta}

Visando mostrar a viabilidade econômica do emprego do wetland construído para o tratamento do efluente doméstico, foi elaborado um cenário aplicando-se algumas formas de redução de custo à ETE experimental. Neste cenário foram mantidas as características principais dela, sendo um wetland de fluxo vertical e com recirculação do efluente do WCFV ao TS, plantado com Heliconia psittacorum. No intuito de reduzir o custo com a aquisição da macrófita, foi considerada a utilização de uma espécie local, como a Canna indica, um material impermeabilizante de baixo custo, o módulo do WCFV escavado no solo e mão de obra local para a execução de alguns serviços.

\section{Cenário 3: ETE fictícia com sistema híbrido}

A configuração de WCFV com recirculação tem como objetivo fornecer condições para que ocorra o processo de desnitrificação do efluente. Outra maneira de se obter a desnitrificação do efluente em sistemas formados por wetlands construídos é a associação em série de uma unidade com fluxo vertical com outra de fluxo horizontal (WCFH), ou seja, WCFV seguido de WCFH, chamado de sistema híbrido. A configuração da primeira unidade favorece a nitrificação do efluente devido à entrada de oxigênio no material filtrante por meio do arraste de oxigênio em cada batelada. Já a segunda unidade oferece as condições anóxicas necessárias para a ocorrência do processo de desnitrificação. Dessa forma, utilizando-se essas duas unidades, não é necessária a recirculação do efluente do WCFV, o que evita o bombeamento. Diante disso, foi feito o dimensionamento de uma unidade fictícia de WCFV para associar com uma unidade de WCFH. Optou-se por utilizar um TS como tratamento primário do efluente. Para o WCFV, assumiu-se que o dimensionamento foi feito com base na taxa de aplicação hidráulica de $120 \mathrm{~mm} \mathrm{~d}^{-1} \mathrm{e}$ vazão de $1.500 \mathrm{~L} \mathrm{~d}^{-1}$. Como material de recheio foram consideradas as britas 1 e 2. Para o dimensionamento do WCFH utilizou-se o critério empírico de relação área/pessoa $\left(\mathrm{m}^{2}\right.$ pessoa $\left.^{-1}\right)$, o qual sugere a área necessária para o reator em um intervalo de 1 a $5 \mathrm{~m}^{2}$ pessoa $^{-1}$ (PHILIPPI; SEZERINO, 2004). Considerou-se como material de recheio do WCFH a areia média. Em ambos os sistemas considerou-se o emprego de uma macrófita local, a planta Canna indica, assim como no cenário 2 .

\section{Custo do ciclo de vida}

O CCV foi calculado por meio do valor total com a implantação, operação, manutenção e desativação.

A estimativa do custo da implantação foi efetuada por meio da elaboração de uma planilha de custos com serviços e materiais necessários na execução. A quantificação de cada item listado foi feita utilizando-se os valores de produtividade variável existentes nas Tabelas de Composições de Preços para Orçamentos (TABELA..., 2010). Para calcular o custo de cada serviço identificado, foi utilizada a tabela Sinapi (CAIXA..., 2019). Os valores para aquisição das bombas centrífugas foram cotados com empresas especializadas no setor de saneamento. Os custos referentes à operação são basicamente os gastos energéticos devido ao acionamento das bombas. Os gastos com energia elétrica foram estimados a partir dos dados de potência de cada bomba centrífuga operante e da tarifa de consumo da companhia de energia elétrica local na modalidade residencial.

Na manutenção foi considerada a substituição do conjunto motobomba, poda das macrófitas e remoção de espécies invasoras. Esses serviços foram considerados com frequência anual, feitos mediante a contratação de um jardineiro, sendo necessária uma média de 3 h de trabalho em um único dia (o valor da hora trabalhada foi obtido pela tabela Sinapi). Foi determinada a substituição das bombas centrífugas a cada 5 anos como forma de manutenção preventiva.

Os custos de desativação envolvem a coleta e o transporte do resíduo e a disposição do material. Para a coleta e o transporte do resíduo foi considerado o uso de caminhão basculante, e o valor de seu aluguel foi obtido pela Sinapi (CAIXA..., 2019). Em relação à disposição do material, obteve-se em consulta a um aterro industrial o valor do metro cúbico para resíduo Classe II-B.

\section{Metodologia da análise do custo do ciclo de vida}

Foi calculada a ACCV pelo método do valor presente líquido (VPL) apresentado na Equação 1 (BLANK; TARQUIN, 2008). O VPL calcula a atratividade dos investimentos da organização, sendo a soma dos valores presentes dos fluxos estimados de uma aplicação, calculados a partir de uma taxa dada e de seu período de duração, e permite que seja feita uma análise sobre a viabilidade de determinado projeto. Dessa forma, podese definir se é rentável ou não (DIMURO et al., 2014). Com a estimativa dos custos de implantação e futuros, estes foram convertidos para uma data-base no presente, ano de 2019, adotando-se a inflação anual média do 
índice de preços IGP-M como taxa de juros, e foi realizado o somatório de todas as despesas no ano-base, obtendo-se um único valor.

$\mathrm{ACCV}=\sum_{\mathrm{t}=0}^{\mathrm{N}} \frac{\mathrm{CCV}}{(1+\mathrm{d})^{\mathrm{N}}}$,

Em que:

ACCV = custo total no valor presente (R\$);

$\mathrm{CCV}$ = soma de todos os custos envolvidos (R\$);

$\mathrm{N}$ = período considerado (anos); e

d = taxa mínima de atratividade (TMA) (\%a.a.).

Com o auxílio da ferramenta Microsoft Office Excel foram utilizadas as equações 2, 3, 4 e 5, referentes à atualização e à projeção dos custos. Foi calculado o valor futuro (F) e o valor presente (P). Para esses cálculos foi considerada a taxa de inflação anual média do índice de preços IGP-M calculada de 2009 a 2019, de 6,2\% a.a. Todos os custos foram ajustados para o ano-base de 2019. O tempo de vida útil das ETE foi considerado 20 anos, sendo o ano de 2039 como horizonte de projeto.

$\mathrm{F}=\mathrm{P}(1+\mathrm{i})^{\mathrm{n}}$

$\mathrm{F}=\mathrm{A}\left[\frac{(1+\mathrm{i})^{\mathrm{t}^{-1}}}{\mathrm{i}}\right]$

$\mathrm{P}=\mathrm{A}\left[\frac{(1+\mathrm{i})^{\mathrm{t}^{-1}}}{\mathrm{i}(1+\mathrm{i})^{\mathrm{t}}}\right]$

$\mathrm{P}=\frac{\mathrm{F}}{(1+\mathrm{i})^{\mathrm{t}}}$

Em que:

$\mathrm{i}=$ inflação (\% a.a.);

$\mathrm{t}$ = período considerado (anos);

$\mathrm{A}$ = série de pagamentos uniformes que se inicia no período 1 e termina no período n (R\$);

$\mathrm{F}=$ valor futuro (R\$); e

$\mathrm{P}=$ valor presente $(\mathrm{R} \$)$.

\section{Resultados e discussão}

\section{Custo do ciclo de vida: cenário 1 (ETE experimental)}

Estimativa do custo da implantação

Na Tabela 1 foram apresentados os custos de implantação estimados para o cenário 1.

O custo de implantação calculado para a ETE experimental no ano de 2019 foi de R \$ 2.566,12 por habitante. Esse custo reflete principalmente os custos com os equipamentos e com o material de recheio. Somados, ambos correspondem a um percentual de $60 \%$ do valor total de implantação. Na implantação o desnível do terreno onde está localizada a ETE experimental não permitiu que o módulo do wetland construído fosse escavado no solo. Assim, foi necessário construir o filtro em alvenaria acima do nível do solo. Para garantir que não ocorressem infiltrações, foi feita uma impermeabilização de maior custo, com manta asfáltica, ao invés de utilizar lona.

No item material de recheio sobressai o custo com a macrófita empregada no sistema. Foram gastos R\$ 2.5466,12 com a compra das mudas da espécie Heliconia psittacorum, um total de 77\% de todo o valor desse item. Em relação às macrófitas adquiridas, foi escolhida essa espécie devido a seu potencial de tratamento, à boa adaptação em wetlands de fluxo subsuperficial com brita e ao embelezamento paisagístico proporcionado (BRIX; SCHIERUP; ARIAS, 2007). Essa espécie ornamental fornece também um estímulo para que a população tenha maior interesse na operação e na manutenção da boa aparência do sistema (KONNERUP; KOOTTATEP; BRIX, 2009). 
Tabela 1 - Custo de implantação do cenário 1 (ETE experimental)

\begin{tabular}{l|l|r}
\hline \multicolumn{1}{c|}{ Serviço } & \multicolumn{1}{c}{ Descrição } & Valor (R\$) \\
\hline Fundação e pilares & $\begin{array}{l}\text { Laje de concreto armado maciça moldada in loco, } \\
\text { espessura de } 10 \mathrm{~cm} \text {, fck=30 MPa; concreto e } \\
\text { armadura para execução dos pilares }\end{array}$ & $3.352,73$ \\
\hline Alvenaria & $\begin{array}{l}\text { Alvenaria de vedação com tijolos maciços } \\
\text { cerâmicos 10,5x5,5x21,5 cm, juntas de 1 cm }\end{array}$ & $1.365,09$ \\
\hline Impermeabilização & Impermeabilização com manta asfáltica & $2.187,80$ \\
\hline Revestimento & Emboço, chapisco e reboco & 889,51 \\
\hline Tubulação e conexões hidráulicas & Tubos e conexões em PVC, perfil metálico & $1.979,14$ \\
\hline Equipamentos & Quatro bombas centrífugas de 3/4 CV & $11.150,00$ \\
\hline Material de recheio do reator & Britas 1 e 2, mudas das macrófitas & $4.022,80$ \\
\hline Instalações elétricas & Instalações para duas bombas & 714,16 \\
\hline & \multicolumn{1}{c|}{ Total } & $25.661,23$ \\
\hline
\end{tabular}

\section{Estimativa do custo de operação, manutenção e desativação}

Com base nas informações fornecidas pelo fabricante das duas bombas centrífugas foi possível calcular a potência instalada na ETE experimental e o consumo anual de energia esperado. Para o cálculo do consumo anual foi considerado o tempo em que as duas bombas operam por dia na ETE, de 16 min por dia, 8 min com a bomba de recalque e $8 \mathrm{~min}$ com a bomba de recirculação. O custo anual foi de $\mathrm{R} \$ 54,18$, considerando a potência instalada de 1,12 kW, a tarifa de consumo de R \$ 0,49 por kW e um consumo anual de 109,01 kW. O consumo energético operacional da ETE experimental ocorre com o acionamento da bomba, feito oito vezes por dia, ficando operante por 1 min em cada acionamento. Embora na operação da ETE haja um considerável número de acionamentos das bombas, isso não causa efeitos de aumento no consumo de energia, pois possui curta duração, não mais que poucos milissegundos, e o motor da bomba da ETE possui potência baixa (3/4 CV). Porém, em ETE maiores, com bombas operando com capacidade superior a $20 \mathrm{CV}$, por exemplo, o impacto desse fator no consumo de energia deve ser considerado.

Após a ocorrência de colmatação não é necessário que a ETE seja encerrada. Essa situação foi considerada somente para a realização da análise de custo do estudo. Nessa situação, há a possibilidade de reposição do meio filtrante, da impermeabilização e das macrófitas do WCFV para a ETE continuar em operação.

Na Tabela 2 foram apresentados os custos referentes à operação, manutenção e desativação da ETE experimental (cenário 1).

O item mais expressivo apresentado na Tabela 2 foi a substituição das bombas, com custo de R\$ 5.575,50, seguido da coleta, transporte e destinação do material filtrante colmatado, de R\$2.924,00. Em relação à projeção dos custos, na operação e manutenção da ETE no ano de 2019 (ano inicial) houve somente os custos com o consumo de energia e com a poda e remoção de espécies forasteiras.

\section{Construção e implantação do sistema experimental}

A primeira etapa da implantação do sistema consistiu em estabelecer o licenciamento ambiental e o projeto executivo. Em relação ao licenciamento ambiental, obteve-se na Secretaria de Proteção Ambiental da Prefeitura Municipal uma dispensa devido à baixa vazão do efluente final no sistema experimental. Não foi necessária a compra da área onde a estação experimental de tratamento de esgoto está localizada, por ter sido cedida pela Universidade Federal de Santa Maria. A área total disponível para o sistema compreende 42 m², a qual foi limpa e compactada antes do início das obras. A etapa de construção do sistema experimental consistiu em construir o TS e o wetland. O WCFV foi dimensionado, tendo sido obtidas as seguintes dimensões: 24,5 $\mathrm{m}^{2}$ de área superficial; e 1,2 $\mathrm{m}$ de profundidade do filtro, sendo 0,75 $\mathrm{m}$ a profundidade útil. A impermeabilização do WCFV foi feita com manta asfáltica, sendo todo o seu interior coberto com uma camada desse material. O wetland construído foi preenchido com 0,5 m de espessura de brita 1 e 0,25 m de brita 2, totalizando-se um volume de $18,375 \mathrm{~m}^{3}$ desse material. O material de preenchimento foi escolhido devido à disponibilidade local e conforme as faixas granulométricas indicadas na literatura especializada. Durante o preenchimento do filtro, que ocorreu com o auxílio de máquina retroescavadeira fornecida pela UFSM, efetuou-se a lavagem de todo o material filtrante. O plantio da macrófita Heliconia psittacorum foi feito diretamente no material de recheio, adotando-se a densidade de 7,7 plantas por metro quadrado, chegando a um total de 188 mudas. Após a construção das unidades, iniciou-se a instalação hidráulica referente à 
colocação das canalizações, conexões (curvas, tês, registro), chaves de boia, bombas e medidores de vazão. A tubulação de distribuição do efluente foi posicionada acima da superfície do WCFV e fixada em suportes de ferro. Essa tubulação é de PVC rígido de $32 \mathrm{~mm}$ de diâmetro com furos de $6 \mathrm{~mm}$ de diâmetro espaçados a cada $10 \mathrm{~cm}$. Ao fundo do reator foi disposta a tubulação de saída/coleta do efluente tratado. Esta é composta de tubos de PVC rígido de $40 \mathrm{~mm}$ de diâmetro com furos de $8 \mathrm{~mm}$ de diâmetro espaçados a cada $10 \mathrm{~cm}$. A tubulação de drenagem foi assentada acima de uma camada de aproximadamente $5 \mathrm{~cm}$ de brita 2 . Em um ângulo de $90^{\circ}$ em relação à tubulação de drenagem se dispôs a tubulação de ventilação do sistema. As instalações elétricas do sistema se referem à parte elétrica dos medidores de vazão e bombas e à rede elétrica. O sistema é composto de quatro bombas centrífugas (duas operando e duas de reserva), uma destinada a recalcar o esgoto do TS para o WFCV, e outra destinada à recirculação do efluente, do filtro para o TS. O sistema conta com três medidores de vazão, sendo dois medidores eletromagnéticos e um ultrassônico.

\section{Desempenho da operação e da manutenção da ETE experimental}

A operação da ETE experimental teve início no dia 10/09/2015. Nesse período, a TAH média no WCFV foi de 52,8 $\mathrm{mm} \mathrm{d}^{-1}$. Inicialmente, no período de 10/09/2015 a 04/01/2016, a aplicação de esgoto no WCFV foi feita com 8 bateladas (pulsos) por dia em um tempo de 2 min por pulso, com um volume médio de $375 \mathrm{~L}$ cada, totalizando $1.500 \mathrm{~L} \mathrm{~d}^{-1}$. Em 05/01/2016 a alimentação do sistema passou a ser realizada por meio de 8 pulsos de 187,5 L cada, totalizando $1.500 \mathrm{~L} \mathrm{~d}^{-1}$. O tempo de cada pulso, controlado por um temporizador, passou a ser de $1 \mathrm{~min}$. O volume de cada pulso no WCFV foi diferente em cada uma das fases operacionais, mas com a mesma taxa de aplicação superficial diária, de $61,22 \mathrm{~L} \mathrm{~m}^{-2} \mathrm{~d}$. Em ambas as fases o tempo de retenção hidráulica de cada pulso foi de $8 \mathrm{~min}$. Durante o período do estudo, os medidores de vazão instalados no sistema realizaram a leitura do volume que passava no sistema. Obteve-se uma vazão afluente média de 1.491 $\mathrm{L} \mathrm{d}^{-1}$, o que representa $99 \%$ da vazão de projeto $\left(1.500 \mathrm{~L} \mathrm{~d}^{-1}\right)$. Quanto aos medidores eletromagnéticos, um se encontra instalado após o TS, medindo a vazão afluente ao WCFV, e outro após o WCFV, medindo a vazão de recirculação. O medidor ultrassônico é utilizado para medir a vazão do efluente que passa no canal com vertedor de $60^{\circ}$ no final do tratamento do WCFV. No período de operação da ETE experimental observado neste trabalho (10/09/2015 a 07/04/2016) o sistema ainda não estava operando com a recirculação do efluente. Nesse período as macrófitas apresentaram bom desenvolvimento, o que demonstra que elas tiveram boa adaptação às condições locais. Não foi necessário realizar poda das plantas, porém algumas plantas invasoras tiveram de ser retiradas, procedimento necessário apenas uma vez no período em questão.

\section{Análise de formas de redução de custo do sistema}

Os wetlands construídos podem ser projetados para valorizar esteticamente o ambiente mediante a seleção de espécies de plantas ornamentais. No sistema experimental de tratamento de esgoto do presente estudo foi utilizada a planta ornamental da espécie Heliconia psittacorum, o que ocasionou um considerável acréscimo no custo da implantação. Entretanto, como flores como a helicônia, além da boa eficiência relatada em wetlands construídos, têm grande valor econômico no mercado (KONNERUPP; KOOTTATEP; BRIX, 2009), pode-se vendê-las, ou seja, há possibilidade de renda e, portanto, de amortização dos custos operacional e de manutenção. Para reduzir o custo de aquisição de plantas, por outro lado, é indicada a adoção de outras espécies nativas locais, que chegam a nem ter custo, ainda com as vantagens da facilidade de obtenção e da adaptação ao clima local. Como exemplo cita-se a taboa (Typha sp.), amplamente utilizada em wetlands construídos por ter bom potencial no tratamento de esgotos (VYMAZAL, 2011). Outras espécies variadas apresentam potencial de utilização em sistemas de wetlands construídos de fluxo vertical, tais como beri (Canna indica) (ZHANG et al., 2014; CUI et al., 2010), sombrinha-chinesa (Cyperus alternifolius) (CHENG et al., 2002), caniço (Phragmites australis) (ZHU et al., 2010; ZHANG et al., 2014), capin-tifton 85 (Cynodon dactycon pers.) (MATOS et al., 2010; COTA, 2011), braquiária do brejo (Brachiaria arrecta) (CALIJURI et al., 2009) e papiro (Cyperus papyrus) (ZANELLA, 2008).

Tabela 2 - Custo de operação, manutenção e desativação do cenário 1 (ETE experimental)

\begin{tabular}{l|c}
\hline \multicolumn{1}{c|}{ Item } & Custo (R\$, 2019) \\
\hline Consumo de energia & 54,18 \\
Poda e remoção de espécies forasteiras & 32,94 \\
Substituição das bombas & $5.575,50$ \\
Coleta, transporte e disposição do material filtrante colmatado & $2.924,00$ \\
\hline
\end{tabular}


O valor de implantação do wetland pode ser sensivelmente reduzido em terrenos com o desnível favorecido e a profundidade de lençol freático adequada, em que há a possibilidade de o módulo do WCFV ser escavado no solo. Diversos são os trabalhos que utilizam os módulos escavados no terreno (AVELAR et al., 2015; PELISSARI et al., 2015). Dependendo da situação, pode ser empregado mutirão para reduzir custos, em que os próprios habitantes do local auxiliam no processo de escavação do solo, na colocação da camada impermeabilizante, na lavagem do material filtrante e no plantio das macrófitas. A impermeabilização do wetland pode ser realizada com lona plástica, que é o material impermeabilizante de menor preço encontrado no mercado. Para sua aplicação é necessária uma camada de areia ou manta geotêxtil antes da colocação da camada de brita, o que evita a perfuração da lona, minimizando a contaminação do solo.

\section{Custo do Ciclo de Vida: cenário 2}

Na Tabela 3 está apresentado o custo de implantação estimado para o cenário 2.

Em comparação ao cenário anterior, foi observada considerável redução do custo de implantação, cerca de $30 \%$. Essa diferença ocorre devido a alguns aspectos construtivos: módulo do WCFV escavado no solo (evita a utilização de alvenaria), impermeabilização com material de menor custo (em comparação com a manta asfáltica) e emprego de macrófita nativa disponível nas proximidades (evita a compra de plantas). Nesse cenário foi considerada mão de obra local para a execução dos seguintes serviços: colocação da lona impermeabilizante, lavagem do material filtrante e transplantio da macrófita.

Os custos operacionais e de desativação do cenário 2 estão apresentados na Tabela 4. Nos cenários 1 e 2 não houve alteração significativa no sistema. Dessa forma, esses custos somente se diferenciaram no item poda e remoção de espécies forasteiras/invasoras.

No cenário 1, na projeção dos custos foi considerada a macrófita Heliconia psittacorum, espécie ornamental e de lento crescimento, enquanto neste cenário não houve custo com a compra de mudas, pois foi utilizada a macrófita local Canna indica. Entretanto, o custo de manutenção, apresentado na Tabela 4, foi superior ao do cenário anterior devido ao fato de a macrófita escolhida para o cenário ser uma espécie local com crescimento mais rápido, o que exige intervalos de poda mais frequentes. Neste estudo o intervalo de poda considerado foi o de frequência quadrimestral.

\section{Custo Ciclo de Vida: cenário 3}

O dimensionamento das unidades da ETE que compõem o cenário 3 resultou em:

(a) WCFV com brita 1 e brita 2 como material filtrante, área de 12,5 $\mathrm{m}^{2}$ (5,0 m x 2,5 m); e

(b) WCFH com areia média como material filtrante, área de $20 \mathrm{~m}^{2}$ (8,0 m x 2,5 m).

Para a estimativa do custo de implantação foi considerado o WCFV feito de alvenaria e o WCFH escavado no solo. Na Tabela 5 estão apresentados os custos de implantação do cenário 3, composto por TS+WCFV+WCFH.

O cenário 3 apresentou o menor custo de implantação em relação aos demais cenários. Esse custo inferior ocorreu principalmente devido ao menor custo com a aquisição dos seguintes itens: equipamentos, material hidráulico e elétrico. Embora o custo de implantação seja inferior ao dos demais cenários, a área construída do cenário 3 é superior devido ao fato de esta ser composta de mais unidades de tratamento, com dois módulos de wetland construído, sendo um WCFV associado em série a um WCFH, enquanto as demais alternativas são compostas de somente um módulo WCFV. Os custos de operação, manutenção e desativação deste cenário estão apresentados na Tabela 6.

A manutenção do sistema teve um custo superior ao dos demais cenários deste estudo devido à substituição de uma bomba e à poda e remoção das espécies forasteiras, que ocorrem em intervalos quadrimestrais e exigem um tempo maior de serviço. Também, o custo com a desativação deste cenário apresenta um valor superior ao das demais alternativas, pois há maior volume de material filtrante necessário aos dois módulos de wetland construído do sistema, o que resulta em um custo superior para a disposição, coleta e transporte dele. Entretanto, o custo operacional é inferior, pois se refere somente ao consumo energético de uma bomba centrífuga. 
Tabela 3 - Custo da implantação para o cenário 2

\begin{tabular}{l|l|c}
\hline \multicolumn{1}{c|}{ Serviço } & \multicolumn{1}{c|}{ Descrição } & Valor (R\$) \\
\hline Módulo escavado & Aluguel de retroescavadeira com operador & 475,00 \\
Impermeabilização & Lona plástica, colocação de duas camadas de lona & 66,25 \\
Rede de tubos e conexões hidráulicas & Tubos e conexões em PVC, e perfil metálico & $1.979,14$ \\
Tanque séptico pré-moldado & Aquisição do tanque séptico e colocação & $2.748,38$ \\
Equipamentos & Quatro bombas centrífugas de 3/4 CV & $11.150,00$ \\
Material de recheio do reator & Britas 1 e 2 & 842,00 \\
Instalações elétricas & Instalações para duas bombas & 714,16 \\
\hline & Total & $17.974,93$ \\
\hline
\end{tabular}

Tabela 4 - Custo de operação, manutenção e desativação do cenário 2

\begin{tabular}{l|c}
\hline \multicolumn{1}{c|}{ Item } & Custo (R\$, 2019) \\
\hline Consumo de energia & 54,18 \\
Poda e remoção de espécies forasteiras & 95,43 \\
Substituição das bombas & $5.575,00$ \\
Coleta, transporte e disposição do material filtrante colmatado & $2.924,00$ \\
\hline
\end{tabular}

Tabela 5 - Custos da implantação do cenário 3

\begin{tabular}{l|l|c}
\hline \multicolumn{1}{c|}{ Serviço } & \multicolumn{1}{c}{ Descrição } & Valor (R\$) \\
\hline Fundação e pilares do WCFV & $\begin{array}{l}\text { Laje de concreto armado maciça moldada in loco, espessura } \\
10 \text { cm, fck=30 MPa. Concreto e armadura para os pilares }\end{array}$ & $2.235,00$ \\
\hline Alvenaria do WCFV & $\begin{array}{l}\text { Alvenaria de vedação com tijolos maciços cerâmicos } \\
10,5 x 5,5 x 21,5 \text { cm, juntas de 1 cm }\end{array}$ & 477,00 \\
\hline Módulo escavado & Aluguel da retroescavadeira com operador e combustível & 448,95 \\
\hline Impermeabilização & $\begin{array}{l}\text { Impermeabilização com manta asfáltica no WCFV e com } \\
\text { lona no WCFH }\end{array}$ & 1128,00 \\
\hline $\begin{array}{l}\text { Revestimento } \\
\text { Tubulação e conexões } \\
\text { hidráulicas }\end{array}$ & Emboço, chapisco e reboco & 187,97 \\
\hline Equipamentos & Tubos e conexões em PVC, e perfil metálico & $1.198,75$ \\
\hline $\begin{array}{l}\text { Material de recheio do reator } \\
\text { Instalações elétricas }\end{array}$ & $\begin{array}{l}\text { Brita 1, brita 2 (recheio do WCFV) e areia média (recheio do } \\
\text { WCFH) }\end{array}$ & $1.457,08$ \\
\hline & Instalações para duas bombas & 356,35 \\
\hline
\end{tabular}

Tabela 6 - Custo de operação, manutenção e desativação do cenário 3

\begin{tabular}{l|r}
\hline \multicolumn{1}{c|}{ Item } & Custo (R\$, 2019) \\
\hline Consumo de energia & 27,09 \\
Poda e remoção de espécies forasteiras & 190,86 \\
Substituição da bomba & $2.787,50$ \\
Coleta, transporte e disposição do material filtrante colmatado & $3.589,50$ \\
\hline
\end{tabular}

\section{Análise do custo do ciclo de vida}

Na Tabela 7 foram resumidos os custos obtidos para o ciclo de vida das ETE do estudo. Esses valores expressam a inflação adotada. Nesses cenários foi considerado o alcance da mesma eficiência na remoção dos poluentes em todas as ETE. 
Tabela 7 - Resumo do custo do ciclo de vida das ETE

\begin{tabular}{|c|c|c|c|}
\hline Custos do Ciclo de Vida (i=6,2\% a.a.) & ETE cenário 1 (R\$) & ETE cenário $2(\mathrm{R} \$)$ & ETE cenário 3 (R\$) \\
\hline Custos totais de implantação & $25.661,23$ & $17.974,93$ & $13.064,10$ \\
\hline Custos totais de operação & $2.209,63$ & $2.209,63$ & $1.104,81$ \\
\hline Custos totais de manutenção & $49.092,09$ & $51.224,10$ & $31.229,65$ \\
\hline Custos totais de desativação & $8.849,00$ & $8.849,00$ & $11.025,90$ \\
\hline Custo total & $85.811,95$ & $71.408,66$ & $56.424,46$ \\
\hline \multicolumn{4}{|c|}{ Análise custo do ciclo de vida (Valor Presente Líquido - VPL) } \\
\hline & $53.315,15$ & $44.524,63$ & $32.438,30$ \\
\hline
\end{tabular}

Ressalta-se que os valores expostos na Tabela 7 são negativos, ou seja, se trata do investimento total do ciclo de vida dos sistemas. Isso ocorre porque não há retorno monetário que promova um balanço financeiro; somente há saídas em forma de capital. A atratividade do empreendimento não está no retorno financeiro, pois não foi considerado tratamento complementar para o reúso do efluente, mas na mitigação de impactos ambientais devido ao lançamento de menores concentrações de poluentes no corpo receptor.

O CCV da ETE experimental (cenário 1) foi superior em relação às demais alternativas, enquanto o cenário 2, que visou obter formas de redução dos custos aplicáveis à ETE experimental, apresentou CCV apenas 17\% inferior. Essa pequena diferença nos CCV das duas ETE ocorreu porque, embora se tenha menor custo de implantação no cenário 1, houve maiores custos transcorridos ao longo de sua vida útil. Em relação aos custos totais do ciclo de vida desses cenários, o item do cenário 2 que apresentou valor superior em relação ao cenário 1 foi o custo total de manutenção, correspondente a uma diferença de R\$2.132,01, o que representa 82\% do preço de aquisição da macrófita Heliconia psittacorum. Portanto, embora esta apresente elevado custo inicial com a compra das mudas, há, posteriormente, amortização desse custo devido à economia com o serviço de poda e remoção de espécies forasteiras, além da possível venda de flores. O cenário 3 apresentou o menor custo do ciclo de vida em relação às demais alternativas apresentadas, sendo 39\% em relação ao cenário 1 e $27 \%$ em relação ao cenário 2 . O CCV do cenário 3 reflete os menores custos totais da implantação, manutenção e operação do sistema.

Entende-se que o cenário 2 apresentaria desempenho de tratamento similar ao do sistema experimental (cenário 1). As diferenças impostas no cenário 2 (tipo de planta e de impermeabilização, por exemplo) em relação ao cenário 1 visam somente à redução do custo de implantação da ETE e, dessa forma, não comprometem a eficiência de remoção de matéria orgânica carbonácea e de nitrogênio. O cenário 3 também apresentaria desempenho similar ao do sistema experimental, considerando que ambos têm por objetivo promover a remoção de matéria orgânica carbonácea e de nitrogênio (pelos processos de nitrificação e desnitrificação).

Em comparação com outro estudo sobre tratamento com wetland construído, Resende (2018) avaliou os custos de dois sistemas experimentais com capacidade de tratamento de 0,64 $\mathrm{m}^{3}$ e 1,5 $\mathrm{m}^{3}$ por dia, e vida útil de 20 anos, na cidade universitária em São Paulo. O CCV do primeiro sistema TS+WCFV+WCFH foi de R\$ 28.884,20, e o CCV do segundo sistema, composto de TS seguido por um wetland construído de fluxo livre com aeração forçada, um decantador secundário e um WCFV, foi de R\$36.930,46. No presente estudo o CCV dos três cenários foi $\mathrm{R}$ \$ 53.315,15, R \$ 44.524,63 e R 32.438,30 respectivamente, superiores aos valores encontrados pelo autor.

\section{Conclusões}

Os cenários sugeridos neste estudo propõem configurações para a satisfatória remoção da matéria orgânica carbonácea, de sólidos e de nitrogênio, em atendimento à legislação ambiental. A remoção de nitrogênio constitui um avanço em relação às unidades de tratamento unifamiliares, tais como tanque séptico seguido de filtro anaeróbio, unidades projetadas especificamente para a remoção de sólidos em suspensão e matéria orgânica carbonácea.

Os resultados mostraram que o valor presente líquido obtido pela metodologia ACCV para os cenários 1, 2 e 3 foram VPL1>VPL2>VPL3. O cenário 1, de maior VPL, por ser formado por uma estação experimental em escala real em sua implantação, teve custos consideráveis com a impermeabilização e a aquisição de macrófitas ornamentais para facilidade da manutenção do sistema, adaptação ao meio filtrante adotado e embelezamento paisagístico. Os demais cenários, de menores VPL, mostraram que os custos de implantação podem ser reduzidos pela utilização de material impermeabilizante de baixo custo, utilização de macrófita local, mão de 
obra familiar e execução do módulo escavado no solo, sendo, dessa forma, uma via de solução às populações não atendidas por sistemas convencionais de tratamento de esgoto. Além disso, em relação aos custos operacionais obtidos, destaca-se a vantagem de as ETE sugeridas serem um tratamento biológico de efluentes, dimensionadas para se obter satisfatória eficiência na remoção dos poluentes. Assim, não há a necessidade de adição de produtos químicos, o que resulta em um reduzido custo operacional.

A metodologia da ACCV mostrou ser uma ferramenta importante na escolha da alternativa de menor custo. Para futuros estudos recomenda-se analisar o custo-benefício de outros sistemas de tratamento biológico unifamiliares, tais como tanque séptico seguido de filtro anaeróbio, tanque séptico seguido de sumidouro, e reator anaeróbio de manta de lodo (UASB) seguido de filtro anaeróbio. Dessa forma seria possível comparar com as alternativas apresentadas neste estudo e encontrar uma ferramenta que possa auxiliar na escolha da ETE mais adequada em relação ao custo e eficiência desejados.

\section{Referências}

ARAÚJO, R. K. Redução de micropoluentes em sistema de tanque séptico e wetland construído vertical com recirculação. Santa Maria, 2018. 163 f. Tese (Doutorado em Engenharia Civil) Universidade Federal de Santa Maria, Santa Maria, 2018.

ARIAS, L.; BERTRAND-KRAJEWSKI, J.; MOLLE, P. Simplified hydraulic model of French verticalflow constructed wetlands. Water Science \& Technology, v. 70, n. 5, p. 909-916, 2014.

ASSOCIAÇÃO BRASILEIRA DE NORMAS TÉCNICAS. NBR 7229: projeto, construção e operação de sistemas de tanques sépticos. Rio de Janeiro, 1993.

AVELAR, F. F. et al. Agronomic performance of Mentha aquatica cultivated in constructed wetlands subject to different organic loading rates. Engenharia Agrícola, v. 35, n. 2, p. 322-330, 2015.

BLANK, L.; TARQUIN, A. Engenharia econômica. 6. ed. McGraw Hill, 2008.

BRIX, H. How 'green' are aquaculture constructed wetlands and conventional wastewater treatment systems? Water Science and Technology, v. 40, n. 3, p. 45-50, 1999.

BRIX, H.; SCHIERUP, H.; ARIAS, C. A. Twenty years experience with constructed wetland systems in Denmark-what did we learn? Water Science and Technology, v. 56, n. 3, p. 63-68, 2007.

CAIXA ECONÔMICA FEDERAL. SINAPI - Índice da construção civil. São Paulo, 2019. Disponível em: http://caixa.gov.br/poder-publico/apoio-poderpublico/sinapi/. Acesso em: 8 jun. 2016.

CALIJURI, M. L. et al. Tratamento de esgotos sanitários em sistemas reatores UASB/ Wetlands construídas e fluxo horizontal: eficiência e estabilidade de remoção de matéria orgânica, sólidos, nutrientes e coliformes. Engenharia Sanitária e Ambiental, v. 14, n. 3, p. 421-430, 2009.

CHANG, J. et al. Treatment performance of integrated vertical-flow constructed wetland plots for domestic wastewater. Ecological Engineering, v. 44, p. 152-159, 2012.

CHEN, T. Y. et al. Application of a constructed wetland for industrial wastewater treatment: a pilot-scale study. Chemosphere, v. 64, n. 3, p. 497-502, 2006.

COOPER, P. F. et al. Reed beds and constructed wetlands for wastewater treatment. European Water Pollution Control, v. 6, n. 7, p. 49, 1996.

CORBELLA, C.; PUIGAGUT, J.; GARFÍ, M. Life cycle assessment of constructed wetland systems for wastewater treatment coupled with microbial fuel cells. Science of the Total Environment, v. 585, p. 355362, 2017.

COTA, R. S.; VON SPERLING, M.; PENIDO, R. C. S. Tracer studies and hydraulic behaviour of planted and un-planted vertical-flow constructed wetlands. Water Science and Technology, v. 64, n. 5, p. 10561063, 2011.

COTA, R.S. Hidrodinâmica e desempenho de filtros verticais com alimentação em pulso, plantados e não plantados, empregados no tratamento de esgoto sanitário bruto. Belo Horizonte, 2011. 215 f. Tese (Doutorado em Saneamento, Meio Ambiente e Recursos Hídricos) - Universidade Federal de Minhas Gerais, Belo Horizonte, 2011.

CUI, L. et al. Removal of nutrients from wastewater with Canna indica L. under different vertical-flow constructed wetland conditions. Ecological Engineering, v. 36, n. 8, p. 1083-1088, 2010. 
DECEZARO, S. T. et al. Vertical flow constructed wetland planted with Heliconia psittacorum used as decentralized post-treatment of anaerobic effluent in Southern Brazil. Journal of Environmental Science and Health Part A, v. 53, n. 13, p. 1131-1138, 2018.

DIMURO, J. L. et al. A financial and environmental analysis of constructed wetlands for industrial wastewater treatment. Journal of Industrial Ecology, v. 18, n. 5, p. 631-640, 2014.

GLUCH, P.; BAUMANN, H. The life cycle costing (LCC) approach: a conceptual discussion of its usefulness for environmental decision-making. Building and Environment, v. 39, n. 5, p. 571-580, 2004.

HORN, T. B. et al. Constructed wetland and photocatalytic ozonation for university sewage treatment. Ecological Engineering, v. 63, p. 134-141, 2014.

JARDINS FILTRANTES. Temporada de reprise (2012). Reportagem do programa: Cidades e Soluções. Entrevistado: Thierry Jacquet (presidente da empresa Phytorestore); Globo News produções, 2011. $1^{\circ}$ bloco (17min). Disponível em: https://www.youtube.com/watch?v=44xuoigQ2do. Acesso em: 27 abr. 2019.

JORDÃO, E. P.; PESSOA, C. A. Tratamento de esgotos domésticos. 4. ed. Rio de Janeiro: ABES, 2014.

KADLEC, R. H.; WALLACE, S. D. Treatment wetlands. 2. ed. Boca Raton, FL.: CRC Press, 2009.

KALE, N. N.; JOSHI, D.; MENON, R. Life cycle cost analysis of commercial buildings with energy efficient approach. Perspectives in Science, v. 7, p. 452-454, 2016.

KONNERUP, D.; KOOTTATEP, T.; BRIX, H. Treatment of domestic wastewater in tropical, subsurface flow constructed wetlands planted with Canna and Heliconia. Ecological Engineering, v. 35, p. 248-257, 2009.

LANA, L. C. O. et al. Performance of a single stage vertical flow constructed wetland system treating raw domestic sewage in Brazil. Water Science \& Technology, v. 68, n. 7, p. 1599-1606, 2013.

MATOS, A. T. et al. Influência da taxa de carga orgânica no desempenho de sistemas alagados construídos cultivados com forrageiras. Engenharia Sanitária e Ambiental, v. 15, n. 1, p. 83-92, 2010.

PELISSARI, C. et al. Wetlands construídos aplicados no tratamento de efluente de bovinocultura leiteira. Revista Engenharia e Construção Civil, v. 2, n. 2, p. 23-33, 2015.

PELISSARI, C. et al. Wetlands construídos de fluxo vertical empregado no tratamento de efluente de bovinocultura leiteira. Revista Eletrônica de Gestão e Tecnologias Ambientais, v. 1, n. 2, p. 223-233, 2013.

PELISSARI, C. Nitrogen transformation in horizontal and vertical flow constructed wetlands applied for dairy cattle wastewater treatment in southern Brazil. Ecological Engineering, v. 73, p. 307-310, 2014.

PENG, J. et al. Performance of a novel circular-flow corridor wetland toward the treatment of simulated high-strength swine wastewater. Ecological Engineering, v. 49, p. 1-9, 2012.

PHILIPPI, L. S.; SEZERINO, P. H. Aplicação de sistemas tipo wetlands no tratamento de águas residuárias: utilização de filtros plantados com macrófitas. Florianópolis: Ed. do Autor, 2004.

PLATZER, C. Design recommendations for subsurface flow constructed wetlands for nitrification and denitrification. Water Science and Technology, v. 40, n. 3, p. 257-263, 1999.

PLATZER, C.; HOFFMANN, H.; CARDIA, W. O wetland como componente de ecosan- experiências com o uso e dimensionamento no clima subtropical. In: INTERNATIONAL CONFERENCE ON SUSTAINABLE SANITATION: FOOD AND WATER SECURITY FOR LATIN AMERICA, Fortaleza, 2007. Proceedings [...] Fortaleza: ECOSANLAC/IWA, 2007.

PUIGAGUTA, J. et al. Subsurface-flow constructed wetlands in Spain for the sanitation of small communities: a comparative study. Ecological Engineering, v. 30, p. 312-319, 2007.

RAI, U. N. et al. Constructed wetland as an ecotechnological tool for pollution treatment for conservation of Ganga river. Bioresource Technology, v. 148, p. 535-554, 2013.

RESENDE, J. D. Avaliação do ciclo de vida ambiental e econômica de sistemas descentralizados de tratamento de esgoto envolvendo wetlands construídos. São Paulo, 2018. 184 f. Tese (Doutorado em Sustentabilidade) - Universidade de São Paulo, São Paulo, 2018. 
ROUSSO, B. Z; PELISSARI, C.; SEZERINO, P. H. Desempenho de um wetland construído horizontal empregado no tratamento de esgoto doméstico ao longo de 20 anos de operação. In: SIMPÓSIO BRASILEIRO SOBRE WETLANDS CONSTRUÍDOS, 2., Curitiba, 2015. Anais [...] Curitiba: Wetlands Brasil/UTFPR, 2015.

SANTOS, M. O. et al. Influência da saturação de fundo de maciços filtrantes componentes de wetlands construídos verticais aplicados no tratamento de esgoto sanitário. Revista AIDIS de Ingeniería y Ciencias Ambientales, v. 9, p. 303-316, 2016.

SCHOLZ, M. Seasonal assessment of vertical-flow wetlands treating domestic wastewater. Wetlands for Water Pollution Control. 2. ed. cap. 4, p. 389-400, 2016.

SEZERINO, P. H. et al. Constructed wetlands and sand filter applied as onsite post- treatment of anaerobic effluent. Water Practice \& Technology, v. 7, n. 3, 2012 a.

SEZERINO, P. H. et al. Two different layouts of constructed wetlands applied as decentralized wastewater treatment in southern Brazil. In: International Conference Wetlands Systems for Water Pollution Control, 13., Perth, 2012. Anais [...] Perth, Australia: IWA, 2012b.

SEZERINO, P. H. et al. Wetlands construídos empregados no tratamento descentralizado de esgotos. In: Exposição de Experiências Municipais em Saneamento, 18., Uberlândia, 2014. Anais [...] Uberlândia: ASSEMAE, 2014.

SILVEIRA, D. Potencial de remoção de nitrogênio em um único estágio de filtros plantados com macrófitas para o tratamento de esgoto doméstico plantados com macrófitas para o tratamento de esgoto doméstico bruto: aporte da biologia molecular para a compreensão dos processos. Florianópolis, 2015. 251 f. Tese (Doutorado em Engenharia Sanitária e Ambiental) - Universidade Federal de Santa Catarina, Florianópolis, 2015.

SISTEMA NACIONAL DE INFORMAÇÕES SOBRE SANEAMENTO. Diagnóstico dos Serviços de Água e Esgoto 2017. Ministério das Cidades, Secretaria Nacional de Saneamento Ambiental, Brasília: SNSA/MCIDADES, 2017.

TABELA DE COMPOSIÇÃO DE PREÇO. TCPO. 13. ed. 2010.

TREIN, C. M. et al. Tratamento descentralizado de esgotos de empreendimentos comercial e residencial empregando a ecotecnologia dos wetlands construídos. Ambiente Construído, Porto Alegre, v. 15, n. 4, p. 351-367, out./dez. 2015.

VON SPERLING, M. Comparison of simple, small, full-scale sewage treatment systems in Brazil: UASBmaturation ponds-coarse filter; UASB-horizontal subsurface-flow wetland; vertical-flow wetland (first stage of French system). Water Science and Technology, v. 713, p. 329-337, 2015.

VYMAZAL, J. Plants used in constructed wetlands with horizontal subsurface flow: a review. Hydrobiologia, v. 674, p. 133-156, 2011.

VYMAZAL, J. Removal of nutrients in various types of constructed wetlands. Science of the Total Environment, v. 380, p. 48-65, 2007.

VYMAZAL, J. The use constructed wetlands with horizontal sub-surface flow for various types of wastewater. Ecological Engineering, v. 35, p. 1-17, 2009.

VYMAZAL, J.; KRÖPFELOVÁ, L. Wastewater treatment in constructed wetlands with horizontal subsurface flow. Environmental Pollution, v. 14. Czech University of Life Sciences Prague, Faculty of Environmental Sciences. Czech Republic: Springer Science, 2009.

WU, S. et al. Development of constructed wetlands in performance intensifications for wastewater treatment: a nitrogen and organic matter targeted review. Water Research, v. 57, p. 40-55, 2014.

ZANELLA, L. Plantas ornamentais no pós-tratamento de efluentes sanitários: wetlands construídos utilizando brita e bambu como suporte. Campinas, 219 f. Tese (Doutorado) - Faculdade de Engenharia Civil, Arquitetura e Urbanismo; Universidade Estadual de Campinas, Campinas. 2008.

ZHANG, D. et al. Application of constructed wetlands for wastewater treatment indeveloping countries e A review of recent developments (2000-2013): review. Journal of Environmental Management, v. 141, p. 116-131, 2014. 
ZHU, G. et al. Potential roles of anaerobic ammonium and methane oxidation in the nitrogen cycle of wetland ecosystems. Applied Microbiology and Biotechnology, v. 86, n. 4, p. 1043-1055, 2010.

\section{Taise Perondi}

Departamento de Engenharia Sanitária e Ambiental | Universidade Tecnológica Federal do Paraná | Linha Santa Bárbara, s/n, Campus Frederico Beltrão | Frederico Beltrão - PR - Brasil | CEP 85601-970| Tel.: (49) 9921-6295 | E-mail: taiseperondi@gmail.com

\section{Delmira Beatriz Wolff}

Departamento de Engenharia Sanitária e Ambiental | Universidade Federal de Santa Maria | Avenida Roraima, 1000, Cidade Universitária | Centro de Tecnologia, sala 522, 2o andar, Prédio 10 | Santa Maria - RS - Brasil| CEP 97105-900 | Tel.: (55) 3220-9631 | E-mail: delmirawolff@hotmail.com

\section{Samara Terezinha Decezaro}

Departamento de Engenharia e Tecnologia Ambiental | Universidade Federal de Santa Maria | Bloco III, $3^{\circ}$ andar, sala 313, Campus Frederico Westphalen, Linha Sete de Setembro | Frederico Westphalen - RS - Brasil | CEP 98400-000 | Tel.: (55) 99982-4108 | E-mail: samaradecezaro@gmail.com

\section{Ronaldo Kanopf de Araújo}

Departamento de Engenharia Sanitária e Ambiental | Universidade Federal de Santa Maria | Rua dos J asmins, 165, Patronato | Santa Maria - RS - Brasil | CEP 97020-420 | Tel.: (55) 99140-1084 | E-mail: ronaldo.kanopf@gmail.com

\section{Ambiente Construído}

Revista da Associação Nacional de Tecnologia do Ambiente Construído Av. Osvaldo Aranha, 99 - 30 andar, Centro

Porto Alegre - RS - Brasil

$$
\text { CEP } 90035-190
$$

Telefone: +55 (51) 3308-4084

Fax: +55 (51) 3308-4054

www. seer. ufrgs. br/ ambienteconstruido

E-mail: ambienteconstruido@ufrgs.br 